\title{
Nur ausgeschlafen zum Glukosetest
}

\begin{abstract}
Vor einem oralen Glukosetoleranztest sollten die Testkandidaten nicht nur ausreichend gefastet, sondern auch lange genug geschlafen haben. Sonst könnte es sein, dass die Ergebnisse fälschlich positiv ausfallen.
\end{abstract}

US-Forscher untersuchten 1559 Erwachsene ohne Diabetesdiagnose. Gemessen wurden ihre $\mathrm{HbA}_{1 \mathrm{c}}-$ Konzentration sowie die Glukosewerte nach einem einem standardmäßigen oralen 75-g-GlukoseToleranztest (oGTT). Zudem befragten die Forscher die Studienteilnehmer, wie viel sie für gewöhnlich schliefen und wie lange sie in den zwei Tagen vor dem oGTT geschlafen hatten.

Wie sich zeigte, stieg die Wahrscheinlichkeit für erhöhte $\mathrm{HbA}_{1 \mathrm{c}}$-Werte mit sinkender gewöhnlicher Schlafdauer. Signifikant war der Zusammenhang für weniger als sieben Stunden. Die Wissenschaftler untersuchten ferner, wie sich ein kurzer Schlaf unmittelbar vor dem Test bei Personen auswirkt, deren $\mathrm{HbA}_{1 \mathrm{c}}$-Werte im Normalbereich liegen $(<6 \%)$.

\section{Kein Diabetes, trotzdem pathologischer oGTT}

Probanden, deren Schlafzeit während der beiden Tage vor dem oGTT in der Summe elf Stunden nicht überschritt, hatten ein beinahe verdoppeltes Risiko für eine 2-Stunden-Glukose-Konzentration von 140 mg/dl oder mehr (Odds Ratio je nach Kürze des Schlafs 1,8-3,1).

Die Forscher betrachten diese Ergebnisse des oGTT als falsch positiv. Gemeint ist damit, dass der Test positiv ausfällt, obwohl der Getestete nicht an Diabetes erkrankt ist. Andererseits ist das Testergebnis mit Blick auf die Insulinresistenz durchaus korrekt, die Konzentration der 2-Stunden-Glukose also nicht fälschlich, sondern tatsächlich erhöht. Denn verkürzter Schlaf kann die Insulinsensitivität vermindern.

Mangelnder Schlaf vor dem Test kann laut Aussage der Forscher ähnliche Folgen für das oGTT-Ergebnis haben wie eine zu kurze Nüchternphase. Sie räumen ein, die Ergebnisse müssten erst noch in weiteren Studien bestätigt werden. „Bis dahin aber halten wir es für das Mindeste, die Patienten vor einem oGTT nicht nur zu fragen, ob sie ausreichend gefastet, sondern auch, ob sie genügend geschlafen haben."

Bliwise DL et al. Am J Med, online 5. Januar 2017; doi: 10.1016/j.amjmed.2016.12.007

\section{Umstrittener Nutzen}

\section{Pneumonietherapie für Diabetiker: Besser mit Steroid?}

Patienten mit ambulant erworbener Pneumonie profitieren möglicherweise von einer kurzfristigen Kortikosteroidtherapie. Laut einer Studie aus der Schweiz gilt das auch bei einem begleitenden Diabetes.

In einer randomisierten, placebokontrollierten Doppelblindstudie der Universität

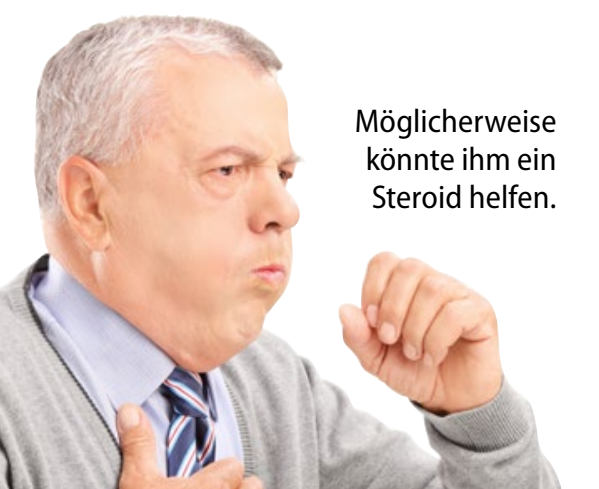

Basel wurde die Wirkung von Kortikosteroiden auf den Verlauf ambulant erworbener Pneumonien (CAP) untersucht. Von den 726 Teilnehmern der Gesamtstudie litten 138 an einem Diabetes; von ihnen erhielten 66 Prednison $(50 \mathrm{mg} / \mathrm{d})$ und 72 Placebo, jeweils für sieben Tage.

\section{Frühere klinische Stabilisierung} Als primärer Endpunkt diente die Zeit bis zur klinischen Stabilisierung (TTCS), definiert als stabile Vitalparameter bei zwei Messungen im Abstand von mindestens zwölf Stunden.

Die TTCS wurde durch Prednison bei Diabetikern signifikant verkürzt (von 6,8 auf 4,5 Tage). Bei allen Prednisonpatienten wurden höhere mittlere Glukosespiegel und mehr Hyperglykämien registriert. Der zusätzliche Insulinverbrauch fiel jedoch nicht höher aus als in der Pla- cebogruppe. Außerdem hatten höhere Glukosespiegel keinen nachteiligen Einfluss auf die untersuchten Endpunkte.

Die European Respiratory Society hat sich bislang noch nicht für den routinemäßigen Einsatz von Kortikosteroiden bei CAP-Patienten ausgesprochen. Die vorliegende Studie wurde u. a. wegen der Wahl des Endpunktes kritisiert. Zwar wurde in einer Metaanalyse ein vermindertes Risiko für die Notwendigkeit einer maschinellen Beatmung und die Entwicklung eines akuten Respiratory-Distress-Syndroms festgestellt. Es gibt jedoch auch Hinweise auf nachteilige Auswirkungen der Kortikosteroidtherapie, z. B. bei viralen Pneumonien. Auch die deutsche Pneumonieleitlinie weist auf diese Unsicherheiten hin.

(bs)

Popovic Met al. Diabetologia 2016;59:2552-60 